\title{
Measurement of normalized differential ttbar cross sections in the dilepton channel from pp collisions at $13 \mathrm{TeV}$ with CMS
}

\author{
Youn Roh*Korea University (KR) \\ E-mail: youn.jung.rohecern.ch \\ on behalf of the CMS Collaboration
}

\begin{abstract}
Normalized differential cross sections for top quark pair production are measured in the dilepton decay channels in proton-proton collisions at a center-of-mass energy of $13 \mathrm{TeV}$. The measurements are performed with data corresponding to an integrated luminosity of $2.2 \mathrm{fb}^{-1}$ using the CMS detector at the LHC. The cross sections are measured differentially as a function of the kinematic properties of the leptons, jets from bottom quark hadronization, top quarks, and top quark pairs at the particle and parton levels. The results are compared to several Monte Carlo generators that implement calculations up to next-to-leading order in perturbative quantum chromodynamics interfaced with parton showering, and also to fixed-order theoretical calculations of top quark pair production up to next-to-next-to-leading order.
\end{abstract}

39th International Conference on High Energy Physics

4-11 July 2018

Seoul, Republic of Korea

\footnotetext{
*Speaker.
} 


\section{Introduction}

Normalized differential cross sections for top quark pair production are measured at particle and parton levels in the dilepton decay channel in proton-proton collisions at a center-of-mass energy of $13 \mathrm{TeV}$ using the CMS experiment [1]. The measurement is made with Run II data recorded in 2015 by the CMS detector corresponding to an integrated luminosity of $2.2 \mathrm{fb}^{-1}$. Simulation data samples are generated by POWHEG, MG5_aMC@NLO and MADGRAPH, showered with either PYTHIA8 or HERWIG++.

\section{Normalized differential cross section measurement}

Normalized differential $t \bar{t}$ cross sections are measured as a function of kinematic variables, $\frac{1}{\sigma} \frac{d \sigma}{d X}$, where $\sigma$ is the total cross section and $X$ represents a variable such as $p_{T}$, rapidity, mass, and $\Delta \phi$ of leptons, top quark and $t \bar{t}$ system. The correction for detector efficiencies, acceptances, and migrations is performed, and the results at particle level are summarized in Figure 1.
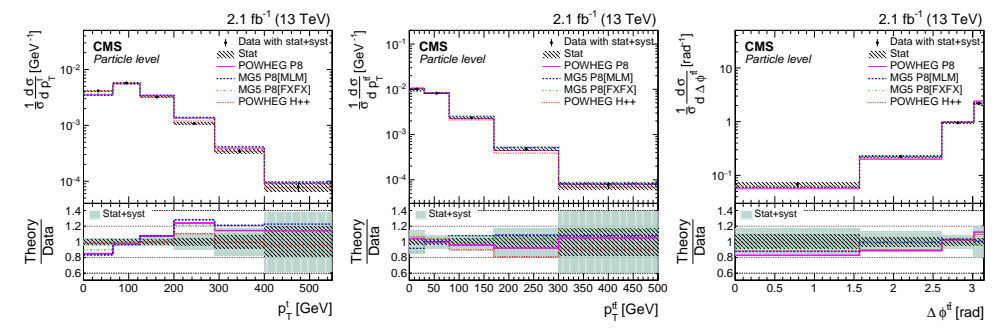

Figure 1: The error bars on data indicate total (combined statistical and systematic) uncertainties, while the dark shaded band shows the statistical uncertainty. The measurements are compared to MC predictions. Figures are taken from [2].

\section{Conclusions}

Normalized differential cross sections of top quark pair production in the dilepton decay channel are measured at particle and parton levels with respect to the leptons, b jets, top quarks, and top quark pairs. The measurements are found to be in agreement with the standard model predictions. More details can be found in [2].

\section{Acknowledgments}

This study was carried out with financial support of the National Research Foundation of Korea (NRF), funded by the Ministry of Science \& ICT under contract NRF-2008-00460. It was also supported by the Basic Science Research Program through the NRF funded by the Ministry of Education (NRF-2016R1A6A3A11933762) and by a Korea University Grant.

\section{References}

[1] CMS Collaboration, "The CMS experiment at the CERN LHC", JINST 3 (2008) S08004

[2] CMS Collaboration, 'Measurement of normalized differential $t \bar{t}$ cross sections in the dilepton channel from pp collisions at 13 TeV”, JHEP04 (2018) 60 